\title{
A Particle Migrating Randomly on a Sphere
}

\author{
David R. Brillinger ${ }^{1}$
}

Consider a particle moving on the surface of the unit sphere in $R^{3}$ and heading towards a specific destination with a constant average speed, but subject to random deviations. The motion is modeled as a diffusion with drift restricted to the surface of the sphere. Expressions are set down for various characteristics of the process including expected travel time to a cap, the limiting distribution, the likelihood ratio and some estimates for parameters appearing in the model.

KEY WORDS: Drift; great circle path; likelihood ratio; pole-seeking; skew product; spherical Brownian motion; stochastic differential equation; travel time.

\section{INTRODUCTION}

There are marine mammals, such as elephant seals, that travel great distances and are tracked. It is of interest to biologists to describe the routes. One can wonder for example if the animals follow great circle paths. The animals will be foraging along the way, i.e., pulled away from the direct route from origin to destination, and this may be modeled as stochastic fluctuations. The great circle route is the geodesic, providing the shortest trip. A ship needs to be changing course continually to stay on it. It is intriguing that some animals apparently do not need to change course, they can keep going straight ahead.

An issue that arises in modeling the physical world is whether to work employing the Itô or the Stratonovich calculus. Reasons have been presented in various places to the effect that, when developing physical applications, it is simpler to start with the Stratonovich form and then switch to the Itô for developing properties of the process. See the discussions in: Bartholdi et al. ${ }^{(19)}$ Karlin and Taylor. ${ }^{(9)}$

To start, some of the previous work on the planar case with drift and the spherical case without drift will be presented.

\footnotetext{
${ }^{1}$ Statistics Department, University of California, Berkeley, California 94720-3860.
} 


\section{THE PLANAR CASE}

Kendall ${ }^{(10)}$ considers the case of a Brownian motion on the plane with an "attractive" polar drift. He works with polar coordinates, $(r, \phi)$, centered at the target center. The particle, in his case a bird, starts at location $(D, 0)$. In a time interval of length $d t$ it moves a distance $\delta d t$ towards the target, then is subject to random Gaussian disturbance, of amount $\sigma d U_{t}$ towards the target and amount $\sigma d V_{t}$ at right angles to the path. Here $U_{t}$ and $V_{t}$ are independent standard Brownian motions and $\sigma^{2}$ their common variance. In Itô form the motion may be described by

$$
\begin{aligned}
& d r_{t}=\left(\frac{\sigma^{2}}{2 r_{t}}-\delta\right) d t+\sigma d U_{t} \\
& d \phi_{t}=\frac{\sigma}{r_{t}} d V_{t}
\end{aligned}
$$

It will be noted later that these equations correspond approximately to motion on a sphere of large radius. The infinitesimal generator of the $r_{t}$ process is

$$
\frac{\sigma^{2}}{2} \frac{d^{2}}{d r^{2}}+\left(\frac{\sigma^{2}}{2 r}-\delta\right) \frac{d}{d r}
$$

Using the criteria developed in Karlin and Taylor ${ }^{(9)}$ or Bhattacharya and Waymire, ${ }^{(3)}$ for this process the point 0 is unreachable, but an entrance point.

Next suppose that there is a circle of radius $a$ about the target, then among the results of the Kendall paper is that the time to get from $(D, 0)$ to the circle has expected value

$$
\left(D-a+\frac{\sigma^{2}}{2 \delta} \log D / a\right) / \delta
$$

This result may be obtained directly from the formulas recorded in Appendix I, with

$$
s(x)=\int_{a}^{x} \frac{1}{z} \exp \left\{z \frac{2 \delta}{\sigma^{2}}\right\} d z
$$

and

$$
m^{\prime}(x)=x \frac{2}{\sigma^{2}} \exp \left\{-x \frac{2 \delta}{\sigma^{2}}\right\}
$$


$x>0$. Here (2.3) is the exponential integral $\operatorname{Ei}(\cdot)$. The naive expression for the expected travel time is $(D-a) / \delta$ corresponding to $\sigma=0$ in $(2.2)$. Kendall also derives an expression for the variance of the travel time.

The invariant distribution of the process is proportional to $m^{\prime}(x)$ above, i.e., a gamma. The likelihood ratio relative to the case $\delta=0$ is

$$
\int_{0}^{T} \frac{1}{\sigma^{2}}(-\delta) d r_{s}-\frac{1}{2} \int_{0}^{T} \frac{1}{\sigma^{2}}\left(\delta^{2}-\frac{2 \delta \sigma^{2}}{r_{s}}\right) d s
$$

following the Cameron-Martin-Girsanov formula recorded in Appendix I. This is maximized by the choice

$$
\hat{\delta}=\frac{1}{T}\left(r_{0}-r_{T}+\sigma^{2} \int_{0}^{T} \frac{1}{r_{s}} d s\right)
$$

When $\delta=0$ the equations considered are the polar coordinate form of Brownian motion in the plane and for $r_{t}$ one has the case $n=2$ of the Bessel process discussed for example in Karlin and Taylor. ${ }^{(9)}$ The transition density function for that process is given there and is

$$
p(t ; q ; r)=g(r) \int_{0}^{\infty} e^{-\lambda_{2} \cdot 2} G(\lambda q) G(\lambda r) g(\lambda) d \lambda
$$

$t>0$ with

$$
g(r)=r, \quad G(r)=\Gamma\left(\frac{3}{2}\right) J_{0}(r)
$$

\section{FORMS OF SPHERICAL BROWNIAN MOTION}

Perrin ${ }^{(15)}$ working from a model of a randomly rotating sphere, determines the density of the angle, $\omega_{t}$, subtended at the center of the sphere between the initial position of a point on the surface and its position $t$ time units later. He finds that density, relative to the measure $\sin \omega d \omega d \phi$ on the sphere is

$$
2 \pi f(\omega, t)
$$

with $f$ satisfying

$$
\frac{\sigma^{2}}{2} \frac{\partial^{2} f}{\partial \omega^{2}}+\frac{\sigma^{2}}{2} \frac{1}{\tan \omega} \frac{\partial f}{\partial \omega}=\frac{\partial f}{\partial t}
$$


Perrin shows the solution of (3.1), with appropriate initial conditions, is

$$
f(\omega, t)=\frac{1}{4 \pi} \sum_{n=0}^{\infty}(2 n+1) P_{n}(\cos \omega) e^{-n(n+1) t \sigma^{2} / 2}
$$

for $t>0$ where $P_{n}$ is the Legendre polynomial of order $n$. Perrin provides some graphs of this function. He also shows that

$$
E \sin ^{2} \omega_{t}=\frac{2}{3}\left(1-e^{-13 \sigma^{2}}\right)
$$

and further remarks that one can get other moments of Legendre polynomials in $\cos \omega$, by integrating them against expression (3.2).

Yosida $^{(23)}$ determines spherical Brownian motion as the unique temporally and spatially homogeneous diffusion process on $S^{2}$. Suppose $\theta$ and $\phi$ denote longitude and colatitude respectively, $0 \leqslant \theta \leqslant \pi$ and $0 \leqslant \phi<2 \pi$. Yosida finds the transition density from the position $(\theta, \phi)$ at time 0 to the position $\left(\theta^{\prime}, \phi^{\prime}\right)$ at time $t$ to be

$$
p\left(t ; \theta, \phi ; \theta^{\prime}, \phi^{\prime}\right)=\sum_{k=0}^{\infty} \sum_{m=-k}^{k} e^{-k\left(k+1{ }^{\prime} t\right.} Y_{k}^{m}(\theta, \phi) Y_{k}^{m}\left(\theta^{\prime}, \phi^{\prime}\right)
$$

Here $Y_{n}^{m}(\theta, \phi)$ is the spherical harmonic

$$
Y_{n}^{m}(\theta, \phi)=e^{i m \phi} P_{n}^{m}(\cos \theta)
$$

for $n=0,1,2, \ldots,|m| \leqslant n$, and $P_{n}^{m}$ is the associated Legendre function and $P_{n}^{0}=P_{n}$, see Terras. ${ }^{(20)}$ The representations (3.2) and (3.3) are seen to correspond when one uses the addition formula for spherical harmonics. Yosida $^{(23)}$ gives the infinitesimal generator of the process as $\sigma^{2} \Delta^{*} / 2$ where $\Delta^{*}$ is the spherical Laplacian

$$
\Delta^{*}=\frac{1}{\sin \theta} \frac{\partial}{\partial \theta}\left(\sin \theta \frac{\partial}{\partial \theta}\right)+\frac{1}{\sin ^{2} \theta} \frac{\partial^{2}}{\partial \phi^{2}}
$$

$0<\theta<\pi$. The $Y_{k}^{m}$ are the eigenfunctions of the operator $A^{*}$, showing one source of the formula (3.3).

The Itô equations for the process are

$$
\begin{aligned}
& d \theta_{t}=\sigma d U_{t}+\frac{\sigma^{2}}{2 \tan \theta_{t}} d t \\
& d \phi_{t}=\frac{\sigma}{\sin \theta_{i}} d V_{t}
\end{aligned}
$$

with $U_{t}$ and $V$, independent Brownians. 
The motion of $\theta$ is what Itô and McKean ${ }^{(8)}$ call the Legendre process on $[0, \pi]$. It has generator

$$
\frac{\sigma^{2}}{2 \sin \theta} \frac{\partial}{\partial \theta}\left(\sin \theta \frac{\partial}{\partial \theta}\right)
$$

Writing

$$
l(t)=\int_{0}^{t} \frac{1}{\sin ^{2} \theta(s)} d s
$$

and with $\psi$ circular Brownian (that is $\sigma B_{l}(\bmod 2 \pi)$, where $B_{l}$ is a Brownian), they show that spherical Brownian may be represented as

$$
[\theta, \phi]=[\theta, \psi(l)]
$$

Stroock $^{(18)}$ works with $\mathbf{Y}$ in $R^{3}$. He obtains the spherical Brownian as the solution of the Itô equation

$$
d \mathbf{Y}=\left(\mathbf{I}-\mathbf{Y} \mathbf{Y}^{\prime} /\left(\mathbf{Y}^{\prime} \mathbf{Y}\right)\right) d \mathbf{B}-\left(\mathbf{Y} /\left(\mathbf{Y}^{\prime} \mathbf{Y}\right)\right) d t
$$

supposing $\mathbf{Y} \neq 0$ and that $\mathbf{B}$ is Brownian $R \rightarrow R^{3}$. In this case the Stratonovich form is

$$
\text { (S) } d \mathbf{Y}=\left(\mathbf{I}-\mathbf{Y} \mathbf{Y}^{\prime} /\left(\mathbf{Y}^{\prime} \mathbf{Y}\right)\right) d \mathbf{B}
$$

(Here and in the following $(S)$ indicates that the Stratonovich form of equation is being employed.) This process stays on the surface and the final term of (3.8) may be thought of as pulling the process back onto the sphere.

With the change to polar coordinates defined by $\mathbf{Y}=(R \sin \theta \sin \phi$, $R \sin \theta \cos \phi, R \cos \theta$ ) expression (3.9) becomes

$$
\text { (S) } d R,=0
$$

(S) $d \theta_{t}=\frac{\sigma}{R_{t}}\left[\cos \theta_{t} \sin \phi_{t} d B_{t}^{1}+\cos \theta_{t} \cos \phi_{t} d B_{t}^{2}-\sin \theta_{t} d B_{t}^{3}\right]$

$$
\text { (S) } d \phi_{t}=\frac{\sigma}{R_{\imath} \sin \theta_{t}}\left[\cos \phi_{t} d B_{t}^{1}-\sin \phi_{l} d B_{t}^{2}\right]
$$

If one sets $\theta_{l}=r_{l} / R$ and takes $R$ large, these become

$$
\begin{aligned}
& \text { (S) } d r_{t} \approx \sigma\left[\sin \phi_{l} d B_{l}^{\prime}+\cos \phi_{l} d B_{l}^{2}\right] \\
& (S) d \phi_{t} \approx \frac{\sigma}{r_{l}}\left[\begin{array}{c}
\left.\cos \phi_{t} d B_{t}^{\prime}-\sin \phi_{t} d B_{t}^{2}\right] \\
77
\end{array}\right.
\end{aligned}
$$


If one converts to the Itô form and $\delta=0$, these become (2.1). In another approach, Oksendal, ${ }^{(14)}$ [pp. 142-143] obtains spherical Brownian as $\mathbf{B} /|\mathbf{B}|$ with a particular time change.

Suppose next that one is focusing on the distance, $\theta_{l}$, to the North Pole. With the change of variables, $X_{t}=\cos \theta_{1},(3.5)$ becomes

$$
d X_{t}=-\sigma^{2} X_{t} d t-\sigma \sqrt{1-X_{t}^{2}} d U_{t}
$$

on $[-1,1]$. This process is considered in Karlin and Taylor, ${ }^{(9)}$ and Matthews. ${ }^{(12)}$ Its infinitesimal generator is

$$
A=\frac{\sigma^{2}}{2}\left(1-x^{2}\right) \frac{d^{2}}{d x^{2}}-\sigma^{2} x \frac{d}{d x}
$$

The eigenfunctions are the Legendre polynomials, $P_{n}(x)$ with eigenvalue $\lambda_{n}=n(n+1)$, see Karlin and Taylor. ${ }^{(9)}$ One sees another connection with (3.2). The so-called scale and speed functions, defined in Appendix I, are

$$
s(x)=\frac{1}{2} \log \frac{1+x}{1-x}
$$

and

$$
m(x)=\frac{2}{\sigma^{2}}\left(x-x_{0}\right)
$$

respectively. The invariant density, proportional to $m^{\prime}(x)$, is the uniform. The points \pm 1 constitute an entrance boundary and are unreachable. Using the expression (A.3) of the Appendix and carrying out the required integrations, the expected time to travel from $x$ to the point $d$ is

$$
\frac{2}{\sigma^{2}} \log \frac{1-x}{1-d}
$$

$-1<x<d<1$.

Roberts and Ursell ${ }^{(16)}$ investigate random walks on the sphere with all directions of movement assumed equally probable. They obtain the formula (3.2) as the limit when the step size gets small and suggest an approximation to distribution of $\omega_{t}$. Hartman and Watson ${ }^{(7)}$ develop various properties of the approximate distribution. [See also Bingham, ${ }^{(4)}$ Watson. $\left.{ }^{(22)}\right]$

\section{THE GREAT CIRCLE CASE}

Suppose that a particle on the sphere is migrating directly towards the North Pole at speed $\delta$ and subject to Brownian disturbances. The North 
Pole is taken for convenience. The Itô differential equations for the process are

$$
\begin{aligned}
& d \theta_{t}=\sigma d U_{t}+\left(\frac{\sigma^{2}}{2 \tan \theta_{t}}-\delta\right) d t \\
& d \phi_{t}=\frac{\sigma}{\sin \theta_{t}} d V_{t}
\end{aligned}
$$

so long as $\theta_{t} \neq 0$ and with $\phi_{t}$ defined $\bmod 2 \pi$. It will be supposed that the particle does not start at $\theta=0$ or $\pi$. (These points are inaccessible.) The equations extend (3.4).

The infinitesimal generator of the process is

$$
\left(\frac{\sigma^{2}}{2 \tan \theta}-\delta\right) \frac{\partial}{\partial \theta}+\frac{\sigma^{2}}{2} \frac{\partial^{2}}{\partial \theta^{2}}+\frac{1}{2} \frac{\sigma^{2}}{\sin ^{2} \theta} \frac{\partial^{2}}{\partial \phi^{2}}
$$

Clearly the process is bounded, simplifying derivations.

Figure 1 shows a simulation corresponding to the application motivating this research. It refers to elephant seals migrating from the California coast into the NW Pacific. One notices the particle meandering once it reaches the neighborhood of its destination, as was to be anticipated. Meandering around the destination may be thought of as the animal foraging there.

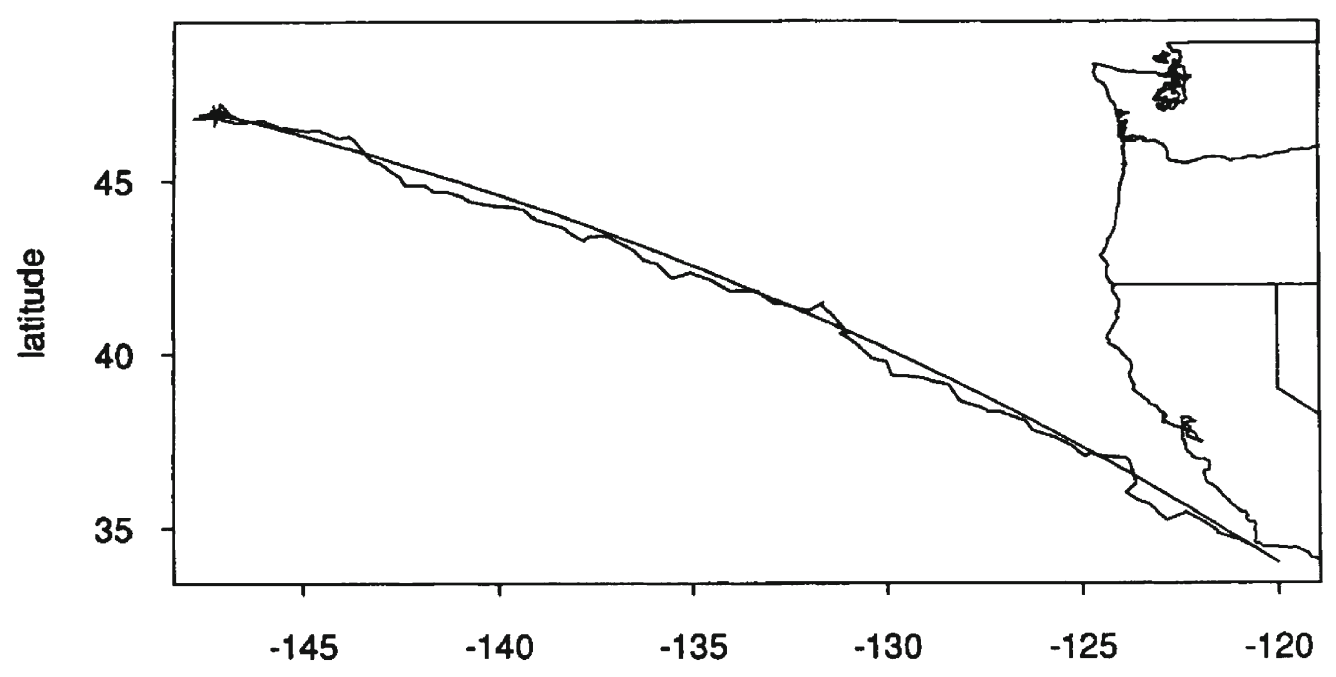

Fig. 1. Simulation of diffusion with drift on sphere. 
With the change of variables $X_{t}=\cos \theta_{t}$, and using Itô's lemma, Eq. (4.1) becomes

$$
d X_{t}=\left(-\sigma^{2} X_{t}+\delta \sqrt{1-X_{t}^{2}}\right) d t-\sigma \sqrt{1-X_{t}^{2}} d U_{t}
$$

whose infinitesimal generator is

$$
B=\frac{\sigma^{2}}{2}\left(1-x^{2}\right) \frac{d^{2}}{d x^{2}}-\left(\sigma^{2} x-\delta \sqrt{1-x^{2}}\right) \frac{d}{d x}
$$

on $[-1,1]$. This reduces to $(3.10)$ when $\delta=0$.

In the case of a sphere of large radius $R$, if one writes $\theta_{t}=r_{t} / R$ one sees that the Eq. (4.1) become

$$
\begin{aligned}
\frac{1}{R} d r_{t} & \approx \sigma d U_{t}+\left(\frac{\sigma^{2}}{2} \frac{R}{r_{t}}-\delta\right) d t \\
d \phi_{t} & \approx \sigma \frac{R}{r_{t}} d V_{t}
\end{aligned}
$$

and replacing $R \sigma$ by $\sigma$ and $R \delta$ by $\delta$ leads back to (2.1). The endpoints of the interval here are inaccessible, but following the general discussion of the topic in Karlin and Taylor ${ }^{(9)}$ can be treated as points of entry. The process is recurrent.

Various characteristics may be derived from the expressions in the Appendix. Following that material one has

$$
I(x, z)=\frac{2 \delta}{\sigma^{2}}\left[\cos ^{-1} x-\cos ^{-1} z\right]-\left[\log \left(1-x^{2}\right)-\log \left(1-z^{2}\right)\right]
$$

The scale function is given by

$$
s(x)=\int_{x_{0}}^{x} \exp \left\{\frac{2 \delta}{\sigma^{2}} \cos ^{-1} z\right\} \frac{1}{1-z^{2}} d z
$$

and the speed function by

$$
m(x)=\frac{1}{\sigma^{2}} \int_{x_{0}}^{x} \exp \left\{-\frac{2 \delta}{\sigma^{2}} \cos ^{-1} z\right\} d z
$$


The invariant density of the process is proportional to

$$
m^{\prime}(x)=\exp \left\{-\frac{2 \delta}{\sigma^{2}} \cos ^{-1} x\right\}
$$

$-1<x<1$. For $\delta / \sigma^{2}$ small, the density is approximately uniform.

Consider next the expected travel time for the process. Suppose the process starts at $x$ and heads to $d, 1>d>x>-1$. Following the expression (A.4) in the Appendix this may be evaluated to

$$
\int_{x}^{d} \frac{2}{\sigma^{2}}\left[\int_{-1}^{y} \exp \left\{-\frac{2 \delta}{\sigma^{2}} \cos ^{-1} z\right\} d z\right] \exp \left\{\frac{2 \delta}{\sigma^{2}} \cos ^{-1} y\right\} \frac{1}{1-y^{2}} d y
$$

In the case that $\delta=0$, it is

$$
\frac{2}{\sigma^{2}} \log \frac{1-x}{1-d}
$$

as given in Section 3.

The skew product representation (3.7) given earlier holds in the present case as well. The proof of Itô and McKean, ${ }^{(8)}$ [p. 200], applies equally.

\section{DETERMINATION OF THE PARAMETERS}

Following the expression (A.5) in Appendix I, the likelihood ratio of the process, relative to that of the case $\delta=0$, is

$$
\frac{1}{\sigma^{2}}\left[(-\delta) \int_{0}^{T} d \theta_{s}-\frac{1}{2} \int_{0}^{T}\left(-\frac{2 \delta \sigma^{2}}{\tan \theta_{s}}+\delta^{2}\right) d s\right]
$$

This leads to the maximum likelihood estimate

$$
\hat{\delta}=\frac{1}{T}\left[\left(\theta_{0}-\theta_{T}\right)+\sigma^{2} \int_{0}^{T} \frac{1}{\tan \theta_{s}} d s\right] / T
$$

Because the particle reaches the region of its destination eventually, this estimate becomes unreasonable as $T \rightarrow \infty$.

One can obtain an exact estimate of $\sigma^{2}$ on the basis of the usual sort of result for quadratic variation

$$
\sum_{i}\left[\tilde{\phi}_{t_{i+1}}-\tilde{\phi}_{t_{i}}\right]^{2} \stackrel{p}{\longrightarrow} \sigma^{2} \int_{0}^{T} \frac{1}{\sin ^{2} \theta_{s}} d s
$$


derived in the Appendix. Here $\left\{t_{i}\right\}$ is a partition of the interval that gets finer under the limiting process, the result is conditional on the (continuous) realization of $\theta_{s}, 0 \leqslant s \leqslant T$, and it is assumed that there exists $\varepsilon>0$ such that $\left|\sin \theta_{s}\right| \geqslant \varepsilon$. The curve $\tilde{\phi}_{\text {, }}$ refers to a continuous curve obtained from the curve $\phi_{t}$ by either patching together continuous segments or by reflecting $\phi_{t}$ whenever it reaches the barriers $\phi=0, \pi$. (It is assumed that $0<\phi_{0}<2 \pi$.) These two constructions are illustrated in Fig. 2. The top
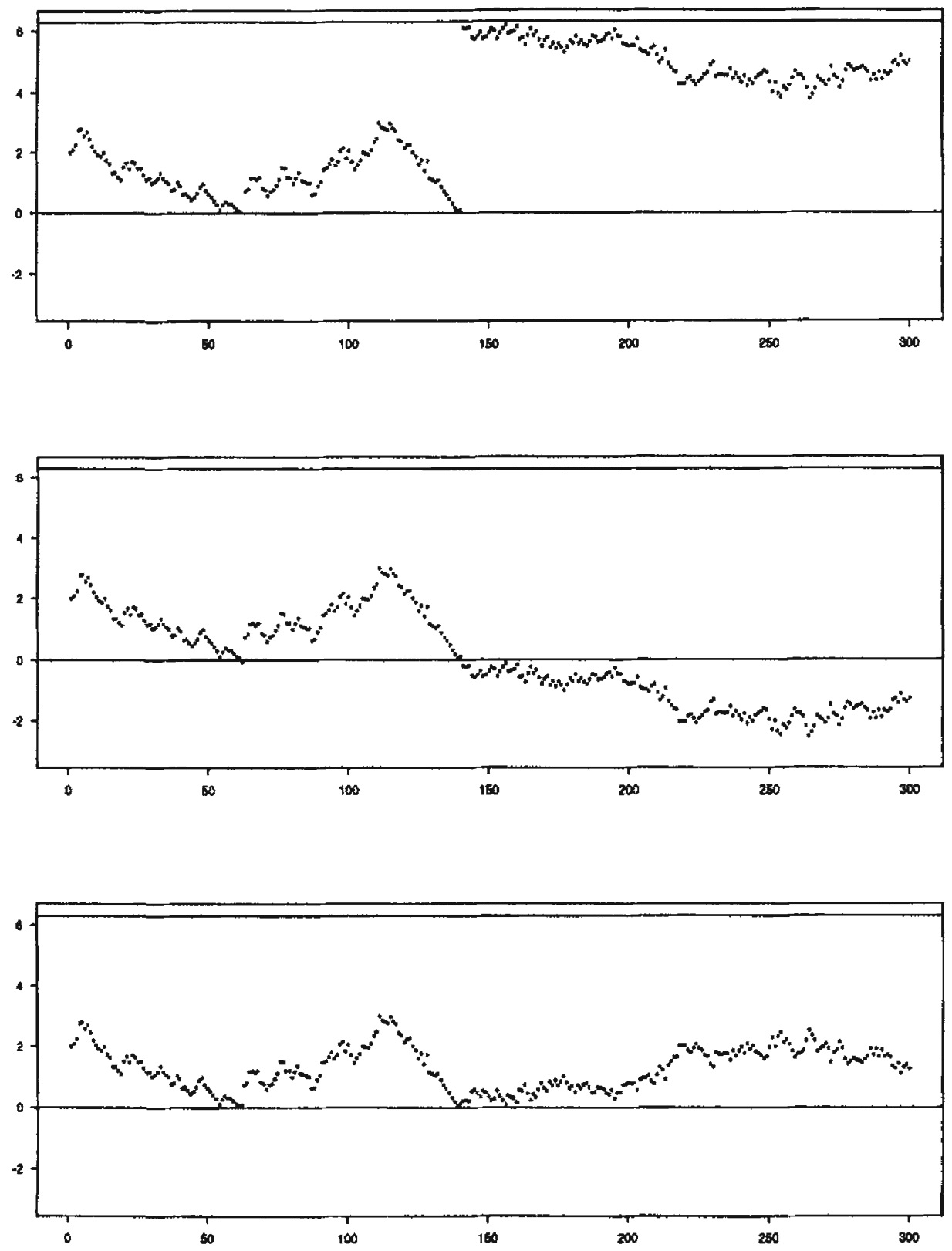

Fig. 2. 
graph represents a realization of $\phi_{t}$. The middle results from joining the two segments continuously and the bottom from reflecting.

When working in practice the data will be available at discrete time points and the above likelihood ratio is not obtainable. However if one has an expression for the transition density, then it can be employed to obtain the likelihood function and estimates of the parameters obtained. An approximate approach here is to do what a ship's navigator has done traditionally. Specifically at the start of a day based on a ship's position the navigator determines the great circle course and that is followed for a day. The next day the navigator determines the ship's new position, again the great circle course based on that position is determined and followed for a day. Unless the ship is heading due north or south, during the course of the days it will be pulled off the great circle route, but with the course revisions the destination is approached. This method leads to approximating the desired transition density by a succession of motions in the plane.

Other approaches to approximating the transition density include: numerical solution of the partial differential equations, some form of quadrature (e.g., that of Dawson ${ }^{(5)}$ ) and simulation.

\section{NAVIGATOR'S COORDINATES}

To obtain the planar approximation just referred to and to prepare Fig. 1 provided earlier, traditional coordinates are helpful. The relations for these are as follows. Let $\phi$ and $\bar{\theta}$ refer to longitude and latitude on the sphere, in radians, $0 \leqslant \phi<2 \pi$ and $-\pi / 2 \leqslant \bar{\theta} \leqslant \pi / 2$. Suppose that a ship is at location $\phi_{t}, \bar{\theta}_{t}$ at time $t$ heading towards position $\Phi, \bar{\Theta}$. The great circle distance, $\rho_{t}$, and course, $\eta_{t}$, satisfy

$$
\begin{aligned}
\cos \rho_{t}= & \cos \left(\frac{\pi}{2}-\bar{\theta}_{t}\right) \cos \left(\frac{\pi}{2}-\bar{\Theta}\right) \\
& +\sin \left(\frac{\pi}{2}-\bar{\theta}_{t}\right) \sin \left(\frac{\pi}{2}-\bar{\Theta}\right) \cos \left(\Phi-\phi_{t}\right) \\
\cos \eta_{t}= & {\left[\cos \left(\frac{\pi}{2}-\bar{\Theta}\right)-\cos \left(\frac{\pi}{2}-\bar{\theta}_{t}\right) \cos \rho_{t}\right] /\left[\sin \left(\frac{\pi}{2}-\bar{\theta}_{t}\right) \sin \rho_{l}\right] }
\end{aligned}
$$

with appropriate choice of quadrant in the latter case. These formulas come from spherical trigonometry and are developed, for example, in Various. ${ }^{(21)}$ 


\section{DISCUSSION}

Corresponding Itô, Stratonovich and differential equation developments have been presented. Each has something to offer and will be employed in the practical study of elephant seal paths in progress.

Another paper working with a diffusion on the sphere, with drift, is Le Gall and Yor. ${ }^{(11)}$ They add general drift terms to Brownian on the sphere and study the equations

$$
\begin{aligned}
& d \theta_{t}=d U_{t}+\frac{1}{2 \tan \theta_{t}} d t+c_{1}\left(\theta_{t}, \phi_{t}\right) d t \\
& d \phi_{t}=\frac{1}{\sin \theta_{t}}\left(d V_{t}+c_{2}\left(\theta_{t}, \phi_{t}\right) d t\right)
\end{aligned}
$$

They obtain asymptotic properties of the windings of the process.

Rogers and Williams ${ }^{(17)}$ develop Brownian motion on submanifolds of $R^{d}$ via a Stratonovich equation. This could be extended to include motion preferring one direction.

\section{APPENDIX}

\section{Appendix I-Some Formulas}

General results for diffusion processes are developed in Gihman and Skorokhod, ${ }^{(6)}$ Karlin and Taylor, ${ }^{(9)}$ and Bhatacharya and Waymire ${ }^{(3)}$ for example. The notation of the latter work is used here.

Consider a diffusion process $X_{l}$ on the line satisfying

$$
d X_{t}=\mu\left(X_{t}\right) d t+\sigma\left(X_{t}\right) d B_{t}
$$

where $B_{t}$ is Brownian motion. Define

$$
I(x, z)=\int_{x}^{z} \frac{2 \mu(y)}{\sigma^{2}(y)} d y
$$

Then the scale function is given by

$$
s(x)=\int_{x_{0}}^{x} \exp \left\{-I\left(x_{0}, z\right)\right\} d z
$$


and the speed function by

$$
m(x)=\int_{x_{0}}^{x} \frac{2}{\sigma^{2}(z)} \exp \left\{I\left(x_{0} z\right)\right\} d z
$$

Following Bhatacharya and Waymire ${ }^{(3)}$ the expected travel time may be written

$$
\int_{x}^{d}(m(y)-m(-1)) d s(y)
$$

if -1 is the lower bound of the state space.

The Cameron-Martin-Girsanov formula for the log likelihood ratio of the process (A.1), relative to the case of $\mu(x)=\mu_{0}(x)$, leads to the expression

$$
\int_{0}^{T} \frac{\left[\mu\left(X_{t}\right)-\mu_{0}\left(X_{t}\right)\right]}{\sigma^{2}\left(X_{t}\right)} d X_{t}-\frac{1}{2} \int_{0}^{T} \frac{\left[\mu^{2}\left(X_{t}\right)-\mu_{0}^{2}\left(X_{t}\right)\right]}{\sigma^{2}\left(X_{t}\right)} d t
$$

See also Gihman and Skorokhod ${ }^{(6)}$ [p. 90].

\section{Appendix II-Determining $\sigma$}

One reference is Basawa and Rao. ${ }^{(2)}$ Consider the quantity

$$
\sum_{i}\left[\tilde{\phi}_{t_{i+1}}-\tilde{\phi}_{t_{i}}\right]^{2}=\sigma^{2} \sum_{i}\left[\int_{t_{i}}^{t_{i+1}} \frac{1}{\sin \theta_{s}} d \tilde{V}_{s}\right]^{2}
$$

Its behavior will be considered conditional on the continuous realization $\left\{\theta_{t}, 0 \leqslant t \leqslant T\right\}$ satisfying $\left|\sin \theta_{i}\right| \geqslant \varepsilon$ for some $\varepsilon>0$.

The expected value of (A.6) is

$$
\sigma^{2} \int_{0}^{T} \frac{1}{\sin ^{2} \theta_{s}} d s
$$

which is $\leqslant \sigma^{2} T / \varepsilon^{2}$. The variance of (A.6) is bounded by $2 \sigma^{4} T$ $\max \left\{t_{i+1}-t_{i}\right\} / \varepsilon^{4}$ which tends to 0 as the partition gets finer. This gives the result. The conclusion is basically a result for the quadratic variation of a martingale.

\section{ACKNOWLEDGMENTS}

It was a privilege to get to work with Murray Rosenblatt early in my career. He set standards of the highest order. I thank him for all the help he has given. 
I have had many enlightening conversations with Brent Stewart concerning the elephant seal data collection program and the behavior of the seals. I thank Ed Ionides, Jim Pitman, and Dan Stoock for useful remarks and I thank Geoff Watson for pointing out the Roberts and Ursell ${ }^{(16)}$ reference.

This paper was prepared with the partial support of the National Science Foundation Grant DMS-9625774 and of the Office of Naval Research Grant N00014-94-1-0042.

\section{REFERENCES}

1. Bartholdi, E., Wokaun, A., and Ernst, R. R, (1976). Theory of stochastic NMR spectroscopy, application of the Itô and Stratonovich calculus. Chem. Phys. 18, 57-84.

2. Basawa, 1. V., and Rao, B. L. S. P. (1980). Statistical Inference for Stochastic Processes. Academic, New York.

3. Bhattacharya, R. N., and Waymire, E. (1990). Stochastic Processess with Applications. John Wiley, New York.

4. Bingham, N. H. (1972). Random walk on spheres. Z. Wahsch. rews. Geb. 22, 169-172.

5. Dawson, D. A. (1981). Galerkin approximation of nonlinear Markov processes. Stutistics and Related Topics. North-Holland, Amsterdam.

6. Gihman, I. I.. and Skorohod, A. V. (1972). Stochustic Differential Equations. Springer, New York.

7. Hartman, P., and Watson, G. S. (1974). "Normal" distribution functions on spheres and the modified Bessel functions. Ann. Prob. 2, 593-607.

8. Itô, K., and McKean, H. P. (1965). Diffusion Processes and Their Sample Paths. Springer, Berlin.

9. Karlin, S., and Taylor, H. M. (1981). A Second Course in Stochastic Processes. Academic, New York.

10. Kendall, D. G. (1974). Pole-seeking Brownian motion and bird navigation. J. Roy. Statist. Soc: 36B, 365-417.

11. Le Gall, J. F., and Yor, M. (1986). Etude asymptotique de certains mouvements browniens complexes avec drift. Prob. Th. Rel. Fields 71, 18.3-229.

12. Matthews, P. (1988). Covering problems for Brownian motion on spheres. Ann. Prob. 16, 189-199.

13. Mortensen, R. E. (1969). Mathematical problems of modelling stochastic nonlinear dynamic systems. J. Stat. Physics 1, 271-296.

14. Oksendal, B. (1995). Stochastic Differential Equations, Fourth Edition. Springer, New York.

15. Perrin, M. F. (1928). Mouvement brownien de rotation. Ann. l'École Norm. Sup. 45, 1-5l.

16. Roberts, P. H., and Ursell, H. D. (1960). Random walk on a sphere and on a Riemanian manifold. Philos. Trans. Roy. Soc. London 252A, 317-356.

17. Rogers, L. C. G., and Williams, D. (1995). Diffusions, Markor Processes and Martingales, Vol. 3. Wiley, New York.

18. Stroock, D. W. (1971). On the growth of stochastic integrals. Z. Wahrsch. verw. Geb. 18, $340-344$.

19. Sussmann, H. J. (1978). On the gap between deterministic and stochastic ordinary differential equations. Ann. Prob. 6, 19-4l. 
20. Terras, A. (1985). Harmonic Analysis on Symmetric Spaces and Applications. Springer, New York.

21. Various (1938). Admiralty Natigation Manual, Vol. III. H. M. Stationery Office, London.

22. Watson, G. S. (1983). Stutistic's on Spheres. Wiley, New York.

23. Yosida, K. (1949). Brownian motion on the surface of the 3-sphere. Ann. Math. Statist. 20 , 292-296. 